\title{
Dynamic admittance of atomic wires
}

\author{
C. C. Wan and José-Luis Mozos \\ Centre for the Physics of Materials and Department of Physics, McGill University, Montreal, Quebec, Canada H3A 2T8 \\ Jian Wang \\ Department of Physics, The University of Hong Kong, Pokfulam Road, Hong Kong \\ Hong Guo \\ Centre for the Physics of Materials and Department of Physics, McGill University, Montreal, Quebec, Canada H3A 2T8
}

(Received 26 February 1997)

\begin{abstract}
We have investigated the low-frequency admittance of quantum wires in which a section consists of several $\mathrm{Al}$ atoms. The atomic section is connected to two three-dimensional leads that are modeled by the jellium model. The quantum scattering problem is solved by combining the first-principles ab initio method with a transfer-matrix evaluation of the scattering matrix. The ac admittance is then computed by evaluating various partial densities of states. The nature of the ac responses are predicted for these $\mathrm{Al}$ atomic wires.
\end{abstract}

[S0163-1829(97)52320-4]

Due to the development of the scanning tunneling microscope (STM) and the atomic force microscope, it is now possible to measure electric conduction through atomic scale objects confined in between a tip and a substrate. ${ }^{1-9}$ Among the interesting results, one important discovery is that the atomic scale tip-substrate contact can have quantized conductance in units of $2 e^{2} / h$, even at room temperature. Transport properties of atomic scale objects can thus have important implications to future quantum electronic applications in addition to the many academic interests. Indeed, free standing nanowires (as opposed to the tip-substrate arrangement) with cross section of about $2 \mathrm{~nm} \times 10 \mathrm{~nm}$ or less have been fabricated, ${ }^{10-13}$ and application of these wires for integrated optoelectronic circuits have been proposed. ${ }^{14}$ A main focus of theoretical investigations of quantum transport through atomic scale objects is to compute the $\mathrm{dc}$ conductance. ${ }^{15,7,6,16,9}$ In this regard, first-principles quantum scattering calculation including the atomic nature of the small contacts has been performed by $\operatorname{Lang}^{17,9}$ for the tipsubstrate geometry, and by the authors for nanowires including three-dimensional (3D) leads. ${ }^{18}$

A very important problem, which has not so far been investigated, concerns the ac transport response of an atomic object. The importance of this problem can be easily realized if we recall the ac properties of various electronic devices, the ac coupling of various units in a circuit, the notion of mesoscopic capacitance, ${ }^{19,20}$ and various theoretical issues concerning ac transport through coherent quantum conductors. The purpose of this work is to report an investigation on the ac transport properties of free standing atomic wires by applying an ac transport formalism developed by Büttiker and co-workers. $19,21,22$

In particular we consider the 3D wire shown in the inset of Fig. 1(a) where an atomic section consisting of several Al atoms is sandwiched between two 3D jellium leads. We assume that each lead is asymptotically a perfect wire that is translationally invariant along the transport direction (the $z$ direction), and the chemical potentials of the leads have a low-frequency oscillating component. An important ac transport formalism has been developed recently that is current conserving and gauge invariant, ${ }^{19,21,22}$ and has been applied to two-dimiensional ballistic conductors by two of the authors. $^{23}$ This formalism relies on the fact that the lowfrequency transport properties of a conductor can be deter-
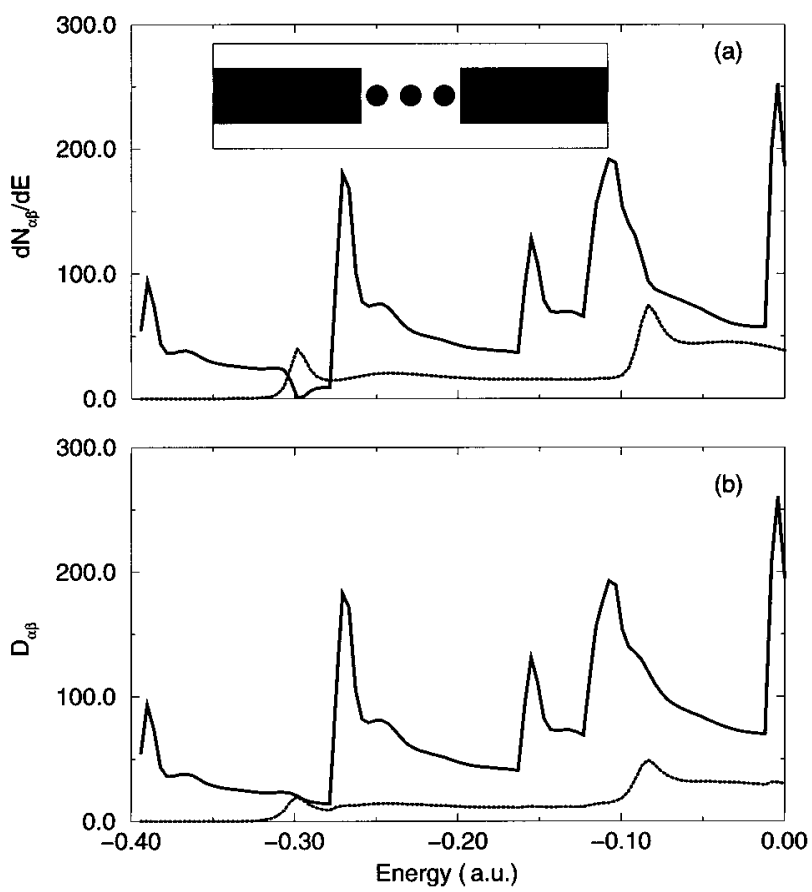

FIG. 1. The partial DOS for the three-atom wire as functions of energy. (a) the GPDOS $d N_{11} / d E$ solid curve; $d N_{21} / d E$ dotted curve. (b) $D_{11}$ solid curve; $D_{21}$ dotted curve. Inset of (a) shows a schematic plot of the quantum wire system: an atomic section with atoms in a chain is sandwiched in between two jellium leads. 
mined by the linear quasistatic electrical response of the conductor to an external potential change, ${ }^{19}$ thus the ac admittance can be calculated from the equilibrium information obtained self-consistently. To linear order in frequency $\omega$, the admittance of a quantum conductor is given by ${ }^{21}$ $g_{\alpha \beta}^{I}(\omega)=G_{\alpha \beta}-i \omega e^{2} E_{\alpha \beta}$, where $G_{\alpha \beta}$ is the dc conductance measured from lead $\beta$ to lead $\alpha$ of the conductor. The superscript $I$ stresses the fact that the emittance matrix $E_{\alpha \beta}$ must be computed by including the electron-electron interaction. ${ }^{21} E_{\alpha \beta}$ can be calculated from the various partial density of states (DOS):

$$
E_{\alpha \beta}=\left(\frac{d N_{\alpha \beta}}{d E}-D_{\alpha \beta}\right)
$$

The first term gives the ac response of the system to the external potential change, and is determined by the global partial density of states (GPDOS)(Ref. 21) $d N_{\alpha \beta} / d E$, which can be computed from energy derivatives of the singleparticle scattering matrix $\left\{s_{\alpha \beta}\right\}$. ${ }^{21}$ This quantity describes the DOS for scattering from lead $\beta$ to lead $\alpha$. The second term of Eq. (1) gives the contribution of the internal potential change induced by the external perturbations, and $D_{\alpha \beta}$ is determined by the local partial density of states (LPDOS) that can be obtained from the scattering wavefunctions. LPDOS is generated by electrons incident from a particular lead regardless where they finally leave the scattering volume. $D_{\alpha \beta}$ has a capacitive nature of the ac response and is equal to the capacitance of the system when there is no dc conduction. To save space we refer interested readers to Refs. 21 for details of this ac transport formalism.

Our calculation proceeds in two steps. ${ }^{18,24}$ First, we calculate the equilibrium properties of the entire wire, including the 3D jellium leads, by applying the well-known density functional theory ${ }^{25}$ and solving the Kohn-Sham equation ${ }^{26}$ to obtain the self-consistent Kohn-Sham electronic wavefunctions $\psi_{i}(\mathbf{r})$ with a self-consistent effective potential $V_{e f f}(\mathbf{r}) \equiv \delta U / \delta \rho(\mathbf{r})$, which is seen by all the electrons. Here $U[\rho]$ is the total self-consistent potential energy, while $\rho$ is the electron density obtained from $\left\{\psi_{i}\right\} .{ }^{26,27}$ We have used the parameterization of Ref. 28 for the exchange-correlation term and a local pseudopotential ${ }^{29}$ for the atomic core. Since we consider cases in which each lead is asymptotically a perfect wire, in our numerical calculations we make sure that the length of the leads is long enough to approximately capture the infinite length limit. Once satisfied, the eigenstates obtained from the equilibrium density-functional calculation can thus be brought into the form of scattering states of the wire. $^{22}$ To directly obtain the scattering states, in the second step of the calculation, we solve a $3 \mathrm{D}$ quantum scattering problem of a particle traversing the wire defined by $V_{\text {eff }}$ (Refs. 30 and 18) to obtain the scattering matrix $\left\{s_{\alpha \beta}\right\}$. Our calculation also gives the scattering wavefunction that is used to compute the LPDOS (Refs. 19 and 23), thus determining $D_{\alpha \beta}$.

At this point we discuss two technical points of our calculation. First, for a finite scattering volume, the GPDOS as determined by the energy derivatives of the scattering matrix $^{21}$ must be corrected in order to guarantee precise current conservation. ${ }^{31}$ In our calculation we shall instead use this conservation law and replace the energy derivatives by a potential derivative. This procedure enables us to accurately determine the GPDOS using quantities local to the finite scattering volume. The validity of this procedure can be proved using Ref. 32, and numerically confirmed in Ref. 33. Hence we computed the scattering matrix $\mathbf{s}=\mathbf{s}\left(V_{o}\right)$ by adding a constant potential $V_{o}$ in the scattering region and performed a three-point numerical derivative of $-d \mathbf{s} /\left.d V\right|_{V_{o} \rightarrow 0}$ to replace $d \mathbf{s} / d E$ which were needed to obtain the GPDOS. ${ }^{32}$ Second, the local response $D_{\alpha \beta}$ can be determined reasonablly easily if the Thomas-Fermi linear screening is used. ${ }^{21,23}$ For the metallic system studied here, we have compared ${ }^{24}$ the dc conductances $G_{\alpha \beta}(E)$ as obtained from the full solution of the Kohn-Sham equation described above and from using a Thomas-Fermi-von Weizsäcker (TFvW) density expanded kinetic-energy functional. ${ }^{27}$ For all the Al wires studied here, we have checked that the two approaches give similar and consistent results for the dc conductances, ${ }^{24}$ providing a justification of using TF to compute the internal responses for the low-frequency admittance calculation.

We have fixed ${ }^{34}$ the jellium leads to have a cross section area of 8.78 a.u. $\times 8.78$ a.u., length $L=23.58$ a.u., and its electron density is specified by the $\mathrm{Al}$ bulk value of $r_{s}=2.07$ a.u. resulting in a total of 90 electrons in the jellium leads. The whole system is put into a supercell of size $16.67 \times 16.67 \times 68.56$ (a.u.) $)^{3}$ for the standard plane-wave based $a b$ initio calculations. ${ }^{26}$ The same jellium density is used for all other wires. A typical potential $V_{\text {eff }}$ profile has been included in Ref. 18. $V_{\text {eff }}$ in the 3D leads is affected by the atoms near the lead-atom junctions, but this effect is essentially damped out away from the junctions. In our selfconsistent calculations of $V_{e f f}$, the numerical convergence is guaranteed within a $\mu \mathrm{eV}$. In the leads, $V_{\text {eff }}$ is essentially a well with a depth $\sim-0.39$ a.u. below the Fermi level in the cross-section plane ( $x, y$ plane) for the three-atom wire. Similar values are obtained for other wires. In the atomic section of the wire, $V_{e f f}$ has rather high peaks due to the ionic core of the atoms. Surrounding the peaks there is the usual attractive part of atomic potential. In the $3 \mathrm{D}$ quantum scattering calculation, we have taken the whole wire, including the leads, as the scattering volume. We have computed the dc conductance of these atomic wires in a previous paper. ${ }^{18}$ For the systems examined, there are six propagating channels in the leads below the Fermi energy. However due to the strong repulsive potential core in the atomic section, only three propagating channels are available there. In this sense the atomic section provides a constriction to the jellium leads and acts as an atomic point $\operatorname{contact}^{35}$ (QPC).

Figure 1(a) shows the external response as given by the GPDOS for an atomic wire with three atoms in the atomic section. Because the transmission of the wire is largely determined by scattering at the atomic section where there are only three channels, the GPDOS for transmission $d N_{21} / d E$ shows "plateaus" similar to that of $G_{21}(E)$. On the other hand, the GPDOS for reflection $d N_{11} / d E$ is determined essentially by the leads where the six channels give rise to the four peaks (in account for degeneracy) below $E_{f} \sim-0.08$ a.u. Indeed, our numerical values of the channel energies are -0.397 a.u., -0.277 a.u., -0.162 a.u., and -0.119 a.u. (the second and fourth are doubly degenerate), 


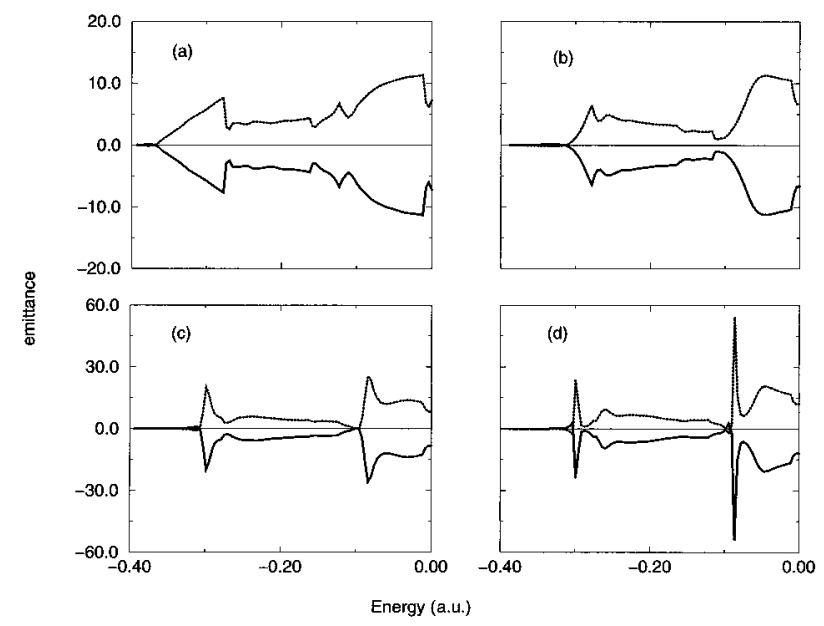

FIG. 2. The dynamic part of the admittance, i.e., the emittance $E_{\alpha \beta}$ as a function of energy. Solid line $E_{11}$; dotted line $E_{21}$. From (a) to (d) are for atomic sections consisting of one, two, three, and four $\mathrm{Al}$ atoms.

which correspond to the peak positions of $d N_{11} / d E$. These peaks reflect the fact that when a transport channel opens, the density of states increases discontinuously. Figure 1(b) shows the internal responses as determined by LPDOS, i.e., the quantity $D_{\alpha \beta}$ of Eq. (1). For this transmissive wire, $D_{\alpha \beta}$ behaves in almost identical fashion as the GPDOS, and this is quite different from situations where transport is mediated by a resonance behavior such as the $T$-shaped junction studied in Ref. 23. At a quantum resonance the electron dwell time takes maximum values, and since the LPDOS measures the time an electron dwells within unit area at a position, as shown previously, ${ }^{23}$ both $D_{11}$ and $D_{21}$ takes maximum values at the resonances with an overall analogous behavior. For our atomic wire studied here, the ac transport is not determined by resonances and $D_{11}$ and $D_{21}$ have completely different energy dependence.

In Fig. 2 we plot the emittance of atomic wires with one, two, three, and four $\mathrm{Al}$ atoms in the atomic section. Several observations are in order. First, we note that all the emittance show spiky shapes as a function of the energy. As discussed above, this is related to the successive opening of higher transmission channels as energy is increased. From Eq. (1) the details of the curves of $E_{\alpha \beta}$ can be understood easily. For example, in Fig. 2(b) the two fast increases of $E_{21}$ at energies $\sim-0.3$ a.u. and -0.1 a.u. are due to the openings of transport channels at the atomic section of the wire. On the other hand the smaller variations of $E_{21}$ in between these two energies are due to the openings of the channels in the leads. Second, an important requirement for low-frequency transport is the current conservation. This requirement demands $\Sigma_{\alpha} E_{\alpha \beta}=0$. Clearly the emittance curves add up to zero in Fig. 2, giving a consistent check to our numerical procedure. Third, from general arguments ${ }^{19}$ we can determine the nature of the low-frequency ac responses of these wires: capacitive if $E_{11}=-E_{21}>0$, or inductive otherwise. Thus the atomic wires studied here all show a dominant inductive ac response, which is expected as the wires are all very transmissive. ${ }^{21}$ However, for longer atomic sections, namely with three or four atoms, the ac responses change nature near the onset of transport channels: Figures 2(c) and 2(d) show small capacitive responses there. Finally, Fig. 2 shows an interesting evolution of the emittance pattern as the number of atoms increases, in particular, the spiky behavior becomes sharper for more atoms. This is consistent with the dc conductance ${ }^{18}$ of these wires where a better quasi-1D behavior is found as the number of atoms in the chain is increased.

Since the atomic section of the wire provides a transport constriction, as mentioned above, the entire wire can be viewed as an imperfect quantum point contact. On the other hand, the ac responses of an ideal QPC (quasi-1D) has been investigated in terms of several transmission parameters, ${ }^{35}$ thus our results can be brought into a similar discussion and there are several similarities and differences. We found that those quantities that are largely determined by the atomic section behave similarly to those of an ideal QPC. Indeed, the spiky behavior of the emittance also exists in an ideal QPC. ${ }^{35}$ The atomic wires show steplike behavior of $D_{21}$, which is the capacitive contribution to the emittance, while the ideal QPC gives a steplike capacitance. ${ }^{35}$ On the other hand, for the atomic wires there are the detailed variations of $E_{\alpha \beta}$ and the peaks of the reflective part of GPDOS and LPDOS. These are largely determined by the transport channels in the leads and were thus absent from the ideal QPC results.

In summary, we have investigated the low-frequency ac responses of $\mathrm{Al}$ quantum wires where there is an atomic section. We have computed, using a first-principles ab initio method combining the density-functional theory with a 3D quantum scattering approach, the various partial density of states and admittance of the atomic wires. The wires studied here are quite transmissive, thus a largely inductive ac response is obtained with some capacitive nature near the onset of transmission subbands for longer atomic sections. The ac responses have a spiky behavior and become stronger and sharper as the atomic section increases. While in this paper we have focused on the emittance of the atomic wires, many interesting further investigations warrant to be made. For example, nontransmissive atomic structures can be established by making the gap between the atom and the leads wider, thus one expects a largely capacitive response. In addition the problem of nonequilibrium charge distribution is very relevant and interesting. ${ }^{35}$ Such a distribution can be investigated using the formalism used here, as shown in Ref. 35, by evaluating the zero-frequency capacitance. Furthermore it is very important to experimentally examine the ac response properties of the atomic systems. Certain predictions of the ac transport theory have already been confirmed by experimental data in the submicrometer regime, ${ }^{36}$ with the STM experimental techniques it will be useful to investigate the atomic regime as well.

H.G. thanks Professor R. Harris and Professor Charles Gale for useful discussions concerning the solution of the 3D quantum scattering problem involved in this paper. We gratefully acknowledge support from NSERC of Canada and FCAR of Québec. J.W. acknowledges support by the Government of Hong Kong under Grant No. HKU 261/95P and the Croucher Foundation. 
${ }^{1}$ J. K. Gimzewski and R. Möller, Phys. Rev. B 36, 1284 (1987).

${ }^{2}$ U. Dürig, O. Züger, and D. W. Pohl, Phys. Rev. Lett. 65, 349 (1990).

${ }^{3}$ N. Agrait, J. G. Rodrigo, and S. Vieira, Phys. Rev. B 47, 12345 (1993).

${ }^{4}$ J. M. Krans, C. J. Muller, I. K. Yanson, Th. C. M. Govaert, R. Hesper, and J. M. van Ruitenbeek, Phys. Rev. B 48, 14721 (1993).

${ }^{5}$ L. Kuipers and J. W. M. Frenken, Phys. Rev. Lett. 70, 3907 (1993).

${ }^{6}$ M. Brandbyge et al., Phys. Rev. B 52, 8499 (1995); L. Olesen et al., Phys. Rev. Lett. 72, 2251 (1994).

${ }^{7}$ J. I. Pascual et al., Science 267, 1793 (1995); Phys. Rev. Lett. 71, 1852 (1993); J. Vac. Sci. Technol. B 13, 1280 (1995).

${ }^{8}$ E. S. Snow, D. Park, and P. M. Campbell, Appl. Phys. Lett. 69, 269 (1996).

${ }^{9}$ Ali Yazdani, D. M. Eigler, and N. D. Lang, Science 272, 1921 (1996).

${ }^{10}$ Hideo Namatsu, Yasuo Takahashi, Masao Nagase, and Katsumi Murase, J. Vac. Sci. Technol. B 13, 2166 (1995).

${ }^{11}$ Dmitri Routkevitch, Terry Bigioni, Martin Moskovits, and Jing Ming Xu, J. Phys. Chem. 100, 14037 (1996).

${ }^{12}$ H. I. Liu, D. K. Biegelsen, F. A. Ponce, N. M. Johnson, and R. F. W. Pease, Appl. Phys. Lett. 64, 1383 (1994).

${ }^{13}$ A. A. Setlur, J. M. Lauerhaas, J. Y. Dai, and R. P. H. Chang, Appl. Phys. Lett. 69, 345 (1996).

${ }^{14}$ Yasuo Wada et al., J. Vac. Sci. Technol. B 12, 48 (1994).

${ }^{15}$ J. A. Torres and J. J. Sáenz, Phys. Rev. Lett. 77, 2245 (1996); Phys. Rev. B 54, 13448 (1996).

${ }^{16}$ R. N. Todorov andA. P. Sutton, Phys. Rev. Lett. 70, 2138 (1993).

${ }^{17}$ N. D. Lang, Phys. Rev. B 52, 5335 (1995).

${ }^{18}$ C. C. Wan, José-Luis Mozos, Gianni Taraschi, Jian Wang, and Hong Guo (unpublished).

${ }^{19}$ M. Büttiker, J. Phys. Condens. Matter. 5, 9361 (1993).

${ }^{20}$ A. Sakai, S. Kurokawa, and Y. Hasegawa, J. Vac. Sci. Technol. A
14, 1219 (1996), and references therein.

${ }^{21}$ For a review of the ac transport formalism, see, for example, M. Büttiker and T. Christen, in Theory of Transport Properties of Semiconductor Nanostructures, edited by E. Schöll (Chapman and Hall, London, 1996).

${ }^{22}$ M. Büttiker, in Quantum Dynamics of Submicron Structures, edited by H. A. Cerdeira, G. Schon, and B. Kramer (Kluwer Academic Publishers, Dordrecht, 1995), pp. 657.

${ }^{23}$ Jian Wang and Hong Guo, Phys. Rev. B 54, R11 090 (1996).

${ }^{24}$ The details of the tedious calculation procedure and other results will be presented elsewhere.

${ }^{25}$ For a review, see, for example, R. G. Parr and W. Yang, Density Functional Theory of Atoms and Molecules (Oxford, New York, 1989).

${ }^{26}$ For a review, see, for example, M. C. Payne et al., Rev. Mod. Phys. 64, 1045 (1992).

${ }^{27}$ N. Govind, José-Luis Mozos, and Hong Guo, Phys. Rev. B 51, 7101 (1995); N. Govind, Jian Wang, and Hong Guo, ibid. 50, 11175 (1994).

${ }^{28}$ S. Goedecker, M. Teter, and J. Hutter, Phys. Rev. B 54, 1703 (1996).

${ }^{29}$ L. Goodwin, R. J. Needs, and V. Heine, J. Phys. Condens. Matter. 2, 351 (1990).

${ }^{30}$ W. D. Sheng and J. B. Xia, Phys. Lett. A 220, 268 (1996).

${ }^{31}$ Jian Wang, Qinrong Zheng, and Hong Guo, Phys. Rev. B 55, 9770 (1997).

${ }^{32}$ M. Büttiker and T. Christen, in Proceedings of Nato Advanced Study Institute on Quantum Transport in Semiconductor Submicron Structures, City, State, year (Kluwer Academic, Bad Lauterberg, Germany, 1995).

${ }^{33}$ Jian Wang, Qingrong Zheng, and Hong Guo, Phys. Rev. B 55, 9763 (1997).

${ }^{34}$ We have used the atomic units throughout this paper.

${ }^{35}$ T. Christen and M. Büttiker, Phys. Rev. Lett. 77, 143 (1996).

${ }^{36}$ W. Chen et al., Phys. Rev. Lett. 73, 146 (1994). 